\title{
Left Ventricular End-Diastolic Pressure and B-Type Natriuretic Peptide Levels Guidance of Low-Dose Furosemide Treatment to Prevent Contrast-Induced Nephropathy in Patients with Percutaneous Coronary Intervention: A Randomized Controlled Trial
}

\author{
Guoqiang Gu $\mathbb{D}$, Demin Liu $\mathbb{D}$, Rui Lu, and Wei Cui \\ Department of Cardiology, The Second Hospital of Hebei Medical University, Shijiahzhuang, Hebei 050000, China \\ Correspondence should be addressed to Wei Cui; cuiweihb2h@163.com
}

Received 29 August 2021; Revised 15 October 2021; Accepted 23 October 2021; Published 5 November 2021

Academic Editor: Jochen Wöhrle

Copyright ( 2021 Guoqiang Gu et al. This is an open access article distributed under the Creative Commons Attribution License, which permits unrestricted use, distribution, and reproduction in any medium, provided the original work is properly cited.

Objective. We aimed to explore the preventive effect of low-dose furosemide administration guided by left ventricular enddiastolic pressure (LVEDP) and B-type natriuretic peptide (BNP) based on adequate hydration on contrast-induced nephropathy $(\mathrm{CIN})$ in patients with percutaneous coronary intervention (PCI). Methods. This parallel randomized clinical trial was conducted at a tertiary hospital in China. A total of 1053 consecutive patients $(71.98 \%$ men) who underwent PCI at our hospital were enrolled. Pre-PCI plasma BNP levels were recorded. Patients enrolled received a continuous intravenous infusion of normal saline starting $4 \mathrm{~h}$ before PCI until $24 \mathrm{~h}$ after surgery. LVEDP was measured immediately after surgery. Patients in the control group received intravenous furosemide injection $(20 \mathrm{mg})$. Patients in the experimental group received furosemide if they showed LVEDP $\geq 15 \mathrm{mmHg}$, a post-PCI BNP level $\geq 100 \mathrm{pg} / \mathrm{mL}$, and/or a post-PCI BNP value $>150 \%$ of the pre-PCI value. The primary and secondary outcome measures were serum creatinine levels, glomerular filtration rate, and creatinine clearance rate measured before and after PCI. CIN incidence was compared between the two groups. Logistic regression analysis was used to study the risk factors for CIN. Results. CIN incidence was significantly higher in the control group than in the experimental group $(P<0.05)$. Logistic regression analysis showed that elevated LVEDP and BNP levels were risk factors. As LVEDP increased, the CIN incidence also increased (odds ratio (OR) 1.038, 95\% confidence interval (CI) 1.006-1.070). The OR of BNP was 1.001 (95\% CI 1.000-1.002). Conclusions. Low-dose furosemide administration guided by LVEDP or BNP is superior to direct low-dose administration on the basis of adequate hydration during PCI. This trial is registered with ChiCTR-IOR-14005250

\section{Introduction}

Contrast-induced nephropathy (CIN) is a common complication of coronary intervention therapy [1]. CIN is defined as an increase in serum creatinine of more than $25 \%$ from the baseline, or a $0.5 \mathrm{mg} / \mathrm{dL}$ increase in the absolute serum creatinine value, within 48 hours after administration of contrast agents. In view of its high incidence and long-term adverse effects, researchers have carried out extensive studies on the prevention measures for CIN [2].
Currently, periprocedural hydration is recognized as an effective strategy for preventing CIN [3]. Moreover, our previous studies have demonstrated that low-dose furosemide administered with adequate hydration reduces the occurrence of CIN more than hydration alone [4]. However, it is crucial to assess the sufficiency of hydration, which requires careful monitoring of the effective blood volume in clinical practice.

Left ventricular end-diastolic pressure (LVEDP) is a reference standard indicator for assessing left ventricular preload and can be used to monitor hemodynamic changes. 
Studies have shown that the volume of hydration treatment can be monitored by measuring the LVEDP during the periprocedural period and the use of diuretics in patients with increased LVEDP levels can significantly reduce the incidence of CIN [5].

In practice, B-type natriuretic peptide (BNP) testing is important for evaluating heart function and correlates with the changing trend of LVEDP, which can identify heart failure and guide treatment [6]. However, it is unknown whether the combined monitoring of BNP and LVEDP levels can guide the use of diuretics to reduce the incidence of CIN. Furthermore, BNP can indirectly reflect the patient's blood volume status. Although BNP testing is not as accurate as LVEDP, BNP measurements are relatively easy to obtain, which is conducive to a wide range of clinical applications. Therefore, our clinical trial attempted to clarify the significance of LVEDP or BNP levels to serve as a guide for the use of low-dose furosemide in a combined hydration treatment for patients who undergo percutaneous coronary intervention (PCI), with the aim of developing an individualized treatment plan for these patients.

\section{Methods}

2.1. Study Patients. A total of 1053 consecutive patients with coronary artery disease (CAD) who underwent PCI were enrolled in this clinical trial (Chinese Clinical Trial Registry ChiCTR-IOR-14005250) that was conducted from October 2017 to May 2019. The study was performed at the Second Hospital of Hebei Medical University. The study protocol conformed to the ethical guidelines of the 1975 Declaration of Helsinki, as reflected in a priori approval by the Ethics Committee of Hebei Medical University (approval number: 2018-R289). All patients provided written informed consent.

2.2. Exclusion Criteria. Patients were excluded for the following reasons: (1) allergic to contrast media, (2) exposure to contrast media within the previous $48 \mathrm{~h}$, (3) kidney or heart transplantation, (4) left ventricular thrombus, (5) congestive heart failure (stage IV), and (6) another severe disease that was a contraindication for hydration.

2.3. Study Design and Experimental Procedures. The patients were randomly assigned to either the experimental or control group. The research staff of the study opened the next sequentially numbered opaque envelope that contained the product assignment. An individual unassociated with the clinical portion of the study prepared the envelopes. The randomization sequence was created using Stata 9.0 (Stata Corp., College Station, TX) statistical software and stratified by the center with a $1: 1$ allocation using random block sizes of 2 , 4 , and 6 . The general characteristics of patients and the results of routine examinations were collected. All patients were treated with $0.9 \%$ sodium chloride solution at a rate of $1 \mathrm{~mL} /$ $\mathrm{kg} / \mathrm{h}$ at $4 \mathrm{~h}$ before surgery until $24 \mathrm{~h}$ after surgery. The BNP value of all patients was measured before surgery, and only the experimental group underwent BNP testing after surgery. The LVEDP was directly measured at the end-diastolic phase using a Cordis pigtail catheter placed inside the left ventricle. The control group was administered an intravenous injection of $20 \mathrm{mg}$ furosemide after PCI. However, the administration of furosemide to those in the experimental group was based on the fulfillment of at least one of the following conditions: LVEDP $\geq 15 \mathrm{mmHg}$, a post-PCI BNP level $\geq 100 \mathrm{pg} / \mathrm{mL}$, and a post-PCI BNP value $>150 \%$ of the pre-PCI BNP value. The full trial protocol can be accessed in the Chinese Clinical Trial Registry (http://www.chictr.org.cn/abouten.aspx) having registration number ChiCTR-IOR-14005250.

2.4. Evaluation Markers. The serum creatinine (SCr) level was measured before and $48 \mathrm{~h}$ after surgery, and the glomerular filtration rate (GFR) and serum creatinine clearance rate $(\mathrm{CCr})$ were calculated as described below. Pre- and postoperative changes in SCr, GFR, and serum CCr values were compared within each group. After the operation, the total fluid intake and urine output at $24 \mathrm{~h}$ were carefully recorded. Other blood sample markers, such as routine blood tests and biochemical parameters, and the incidence of CIN, were also compared between these two groups.

2.5. Diagnostic Criteria. CIN was defined as an elevation in SCr of $>25 \%$ from baseline or $>0.5 \mathrm{mg} / \mathrm{dL}$ within $48 \mathrm{~h}$ of contrast agent administration, with the exclusion of other causes of acute renal injury. The formula for the modification of the diet for renal disease was used to calculate the GFR: GFR $\quad\left(\mathrm{mL} \bullet \mathrm{min}^{-1} \bullet 1.73 \mathrm{~m}^{-2}\right)=186 \times \mathrm{SCr} \quad(\mu \mathrm{mol} /$ $\left.\mathrm{L}^{-1.154}\right) \times$ age $\left(\right.$ years $\left.^{-0.203}\right) \times(0.742$, if female $)$. The Cockcroft-Gault formula was used to calculate the serum CCr : $\mathrm{CCr}(\mathrm{mL} / \mathrm{min})=[140-$ age $($ years $) \times$ weight $(\mathrm{kg}) \times(0.85$ if female $)] /[72 \times \operatorname{SCr}(\mu \mathrm{mol} / \mathrm{L})]$.

2.6. Statistical Analyses. SPSS 21.0 software (IBM Corp., Armonk, NY, USA) was used for the statistical analyses. Measurement data are presented as the mean \pm standard deviation or median and interquartile range. Classification data are presented as percentages. SCr, serum CCr, and GFR values were compared between the two groups using repeated-measure analysis of covariance. The pre- and postsurgical comparisons within each group were conducted using the rank-sum test. The incidence of CIN in all patients or subgroups was analyzed using the $\chi^{2}$ test. Logistic regression analysis was used to determine the risk factors for CIN. A $P$ value of $<0.05$ was considered statistically significant.

2.7. Patient and Public Involvement. It was not appropriate or possible to involve patients or the public in the design, conduct, reporting, or dissemination plans of our research.

\section{Results}

3.1. General Baseline Characteristics of the Participants. A total of 1053 consecutive patients with CAD who underwent PCI were enrolled. Of the 1053 patients, 758 (71.98\%) were men and 295 (28.02\%) were women, with an age range of 
21-84 years (mean, 59.0 years; SD, 13.0 years), a weight range of $42-135 \mathrm{~kg}$ (mean, $70.5 \mathrm{~kg} ; \mathrm{SD}, 15.0 \mathrm{~kg}$ ), and 58 developed CIN. The baseline characteristics of all patients are shown in Table 1.

3.2. Comparison of Baseline Characteristics between the Control and Experimental Groups. There were several significant differences in baseline characteristics between the control and experimental groups, including left ventricular diameter, 24-hour total intake, blood homocysteine level, and C-reactive protein level $(P<0.05$, Table 1$)$.

No significant differences were observed in other general baseline clinical conditions (age, height, weight, sex, and smoking), concomitant diseases (hypertension, diabetes, chronic renal insufficiency, or hyperlipidemia), laboratory indicators (fasting blood glucose, total cholesterol, albumin, urea nitrogen, serum creatinine, uric acid, cystatin $\mathrm{C}, \beta 2$ microglobulin, platelet, and hemoglobin levels), and operative data (number of coronary artery lesions, puncture site, and type/dose of contrast media) $(P>0.05$, Table 1$)$.

3.3. Diagnostic Markers of the Experimental and Control Groups. In the control group, the postoperative SCr levels were significantly higher than the preoperative levels $(P<0.05$, Supplementary Table S1), and the GFR and serum CCr values significantly decreased after surgery $(P<0.05$, Supplementary Table S1).

In the experimental group, the postoperative SCr values decreased slightly from preoperative levels, although the difference was not significant (Supplementary Table S1). The GFR and serum CCr values increased after surgery, although these differences were not significant (Supplementary Table S1).

3.4. Incidence of CIN. The incidence of CIN was significantly higher in the control group than in the experimental group (8.14\% and $2.86 \%$, respectively; $P<0.05$, Table 2 ).

Patients in the control group received intravenous furosemide injection $(20 \mathrm{mg})$. Patients in the experimental group received furosemide if they showed LVEDP $\geq 15 \mathrm{mmHg}$, a post-PCI BNP level $\geq 100 \mathrm{pg} / \mathrm{mL}$, and/or a post-PCI BNP value of $>150 \%$ of the pre-PCI value. The incidence of CIN in subgroups of the experimental group was not significant $(P>0.05$, Table 3$)$.

3.5. Logistic Regression Analysis. Binary logistic regression analysis was performed to further explore the predictive value of LVEDP and BNP for CIN. The results showed that, for LVEDP, the odds ratio (OR) was 1.038, with a $95 \%$ confidence interval $(\mathrm{CI})$ of $(1.006,1.070),(P=0.020)$. For $\mathrm{BNP}$, the OR was 1.001 , with a $95 \%$ CI of $(1.000,1.002)$, $(P=0.021)$. These findings indicate that the incidence of CIN increased with an increase in the LVEDP and BNP values (Supplementary Table S2).

The receiver operating characteristic curve analysis of the predictive performance of LVEDP for CIN produced an area under the curve value of 0.606 , with a sensitivity of $39.2 \%$ and specificity of $77.1 \%(P=0.013)$ (Figure 1$)$. The optimal
LVEDP cut-off for the prediction of CIN was $15.5 \mathrm{mmHg}$. The area under the curve value for BNP was 0.605 , with an optimal cut-off value of $116.5 \mathrm{pg} / \mathrm{mL}$, a sensitivity of $40.0 \%$, and a specificity of $83.7 \%(P=0.014)$ (Figure 1$)$.

\section{Discussion}

We found that under the same hydration plan, LVEDP and BNP measurements in the experimental group were significantly lower than those with the conventional administration of furosemide in the control group. Logistic regression analysis showed that increased LVEDP and BNP levels were positively correlated with the occurrence of CIN. The following reasons potentially contribute to the findings of this study: (1) all patients in the control group were treated with furosemide. However, this hydration scheme may not be sufficient for patients with normal BNP levels and/or LVEDP levels in the normal or low range, which can aggravate renal dysfunction. (2) Patients meeting the conditions of the experimental group had an equivalent increase in volume load, in which the $20 \mathrm{mg}$ of furosemide provided on a hydration-level basis was relatively advantageous. Diuresis can accelerate the excretion of contrast agents and reduce the damage of the contrast agent to the renal tubules.

CIN, a complication associated with PCI, is an important component of iatrogenic renal injury. It has become a vital issue for cardiologists because it may prolong hospitalization and affect long-term prognosis. To our knowledge, the fundamental pathogenesis of CIN is ischemia and hypoxia in the renal medulla. At present, hydration is the only recognized clinical strategy to prevent CIN according to the IIA recommended level in the guidelines [7]. However, due to the limitations of hydration therapy shown in our previous study [5], special attention should be paid to the degree of hydration in clinical practice, especially for patients with heart failure. Notably, hyperhydration may increase the potential for pulmonary edema.

Furosemide administration has a renal protective effect against CIN [8]. Theoretically, furosemide administration attenuates direct toxicity through increased urine flow by enhancing contrast dilution in the renal tubule. Furosemide then inhibits tubular sodium reabsorption in the medulla. Concurrently, furosemide reduces the tubular burden and oxygen requirement, alleviating hypoxia of the ascending medullary branch. The mechanism mentioned above has been verified by the findings of a coauthor's clinical study [4]. Similarly, a clinical meta-analysis of randomized trials has found that furosemide with matched hydration using the RenalGuard system reduced the incidence of contrast-induced acute kidney injury in high-risk patients undergoing PCI or transcatheter aortic valve replacement [9]. The authors suggested that maintaining a high urine output $(>300 \mathrm{~mL} / \mathrm{h})$ during the operation has a direct protective effect on the renal tubular cells. However, some studies with small sample sizes have shown that the administration of prophylactic furosemide may lead to deterioration of renal function after PCI [10]. A meta-analysis conducted in 2015 has indicated that furosemide fails to exert benefits in reducing the incidence of CIN and long-term adverse events after PCI [11]. Therefore, 
TABLE 1: Baseline clinical characteristics of all patients.

\begin{tabular}{|c|c|c|c|}
\hline Factors & Control group & Experimental group & $P$ value \\
\hline Female sex (\%) & $28.60 \%$ & $27.43 \%$ & 0.673 \\
\hline Age (years) & $59.00(13.00)$ & $59.00(12.00)$ & 0.803 \\
\hline Weight (kg) & $70.00(15.00)$ & $71.00(15.00)$ & 0.815 \\
\hline Height $(\mathrm{cm})$ & $170.00(11.00)$ & $169.00(11.00)$ & 0.525 \\
\hline Hypertension, $n(\%)$ & $305(57.77 \%)$ & $309(58.86 \%)$ & 0.719 \\
\hline Diabetes, $n(\%)$ & $141(26.70 \%)$ & $145(27.62 \%)$ & 0.739 \\
\hline Hyperlipidemia, $n$ (\%) & $225(42.61 \%)$ & $240(45.71 \%)$ & 0.311 \\
\hline Smoking, $n(\%)$ & $202(38.48 \%)$ & $208(39.39 \%)$ & 0.760 \\
\hline Statins, $n(\%)$ & $457(86.55 \%)$ & $435(82.86 \%)$ & 0.096 \\
\hline $\mathrm{ACEI} / \mathrm{ARB}, n(\%)$ & $215(40.72 \%)$ & $219(41.71 \%)$ & 0.743 \\
\hline Ejection fraction (\%) & $61.86(3.03)$ & $61.97(2.52)$ & 0.297 \\
\hline Left ventricular diameter (mm) & $48.00(6.00)$ & $47.00(6.00)$ & 0.021 \\
\hline LVEDP (mmHg) & $15.00(9.00)$ & $15.00(8.00)$ & 0.923 \\
\hline BNP before PCI (pg/mL) & $31.60(67.70)$ & $30.30(61.00)$ & 0.677 \\
\hline Total input in $24 \mathrm{~h}(\mathrm{~mL})$ & $4250.66 \pm 1006.65$ & $4141.35 \pm 1022.26$ & 0.046 \\
\hline Total output in $24 \mathrm{~h}(\mathrm{~mL})$ & $4539.96 \pm 1092.81$ & $4521.02 \pm 1838.11$ & 0.178 \\
\hline Fasting blood glucose $(\mathrm{mmol} / \mathrm{L})$ & $4.98(1.56)$ & $5.06(1.58)$ & 0.498 \\
\hline Total cholesterol $(\mathrm{mmol} / \mathrm{L})$ & $4.07(1.22)$ & $4.08(1.39)$ & 0.212 \\
\hline Albumin $(\mathrm{g} / \mathrm{L})$ & $40.90(5.30)$ & $41.40(4.90)$ & 0.090 \\
\hline Creatinine (umol/L) & $69.45(17.33)$ & $69.30(18.80)$ & 0.772 \\
\hline Uric acid (umol/L) & $298.00(105.00)$ & $304.00(110.00)$ & 0.701 \\
\hline Cystatin C (mg/L) & $0.97(0.30)$ & $0.96(0.33)$ & 0.878 \\
\hline Macroglobulin (mg/L) & $1.50(0.72)$ & $1.54(0.67)$ & 0.213 \\
\hline Platelets $\left(10^{9} / \mathrm{L}\right)$ & $206.00(71.00)$ & $204.00(68.00)$ & 0.619 \\
\hline Hemoglobin $(\mathrm{g} / \mathrm{L})$ & $138.00(18.00)$ & $138.00(18.00)$ & 0.684 \\
\hline Homocysteine (umol/L) & $13.95(11.93)$ & $12.95(11.93)$ & 0.016 \\
\hline C-reactive protein $(\mathrm{mg} / \mathrm{L})$ & $3.00(4.58)$ & $2.40(3.58)$ & 0.007 \\
\hline Iopromide, $n(\%)$ & $454(85.98 \%)$ & $470(89.52 \%)$ & 0.080 \\
\hline Contrast agent dose (mL) & $120.00(60.00)$ & $100.00(60.00)$ & 0.197 \\
\hline Right radial artery, $n(\%)$ & $451(85.90 \%)$ & $457(87.05 \%)$ & 0.443 \\
\hline Acute myocardial infarction, $n(\%)$ & $195(36.93 \%)$ & $186(35.43 \%)$ & 0.612 \\
\hline \multicolumn{4}{|c|}{ Severity of coronary artery disease, $n(\%)$} \\
\hline Single branch & $258(48.86 \%)$ & $237(45.14 \%)$ & 0.354 \\
\hline Double branch & $157(29.73 \%)$ & $158(30.10 \%)$ & 0.898 \\
\hline Three branches & $113(21.40 \%)$ & $130(24.76 \%)$ & 0.196 \\
\hline
\end{tabular}

ACEI/ARB, angiotensin-converting enzyme inhibitors/angiotensin receptor blockers; BNP, B-type natriuretic peptide; LVEDP, left ventricular end-diastolic pressure; PCI, percutaneous coronary intervention.

TABLE 2: Incidence of contrast-induced nephropathy in the control and experimental groups.

\begin{tabular}{lccc}
\hline Group & Patients receiving furosemide $(n)$ & CIN & Incidence $(\%)$ \\
\hline Control $(n=528)$ & 528 & 43 & 8.14 \\
Experimental $(n=525)$ & 381 & 15 & 2.86 \\
\hline
\end{tabular}

CIN, contrast-induced nephropathy.

TABLE 3: Incidence of CIN in different subgroups within the experimental group.

\begin{tabular}{|c|c|c|c|c|}
\hline Group & Patients treated with furosemide $(n)$ & $\mathrm{CIN}$ & Incidence (\%) & $P$ value \\
\hline $\operatorname{BNP}^{*}(n=78)$ & 78 & 3 & 3.85 & \\
\hline $\operatorname{LVEDP}^{\hat{\imath}}(n=153)$ & 153 & 4 & 2.61 & 0.95 \\
\hline $\mathrm{BNP}^{2} \operatorname{LVEDP}^{\odot}(n=15)$ & 150 & 4 & 2.67 & \\
\hline Incompatible with both standards $(n=144)$ & 0 & 4 & 2.78 & \\
\hline
\end{tabular}

BNP, B-type natriuretic peptide; CIN, contrast-induced nephropathy; LVEDP, left ventricular end-diastolic pressure. Patients in the experimental group received furosemide if they showed LVEDP $\geq 15 \mathrm{mmHg}$, a post-PCI BNP $\geq 100 \mathrm{pg} / \mathrm{mL}$, and/or a post-PCI BNP value of $>150 \%$ of the pre-PCI value. ${ }^{*}$ Use of furosemide only met the BNP standard; "Use of furosemide only met the LVEDP standard; ${ }^{\circ}$ Use of furosemide met the BNP and LVEDP standards. 


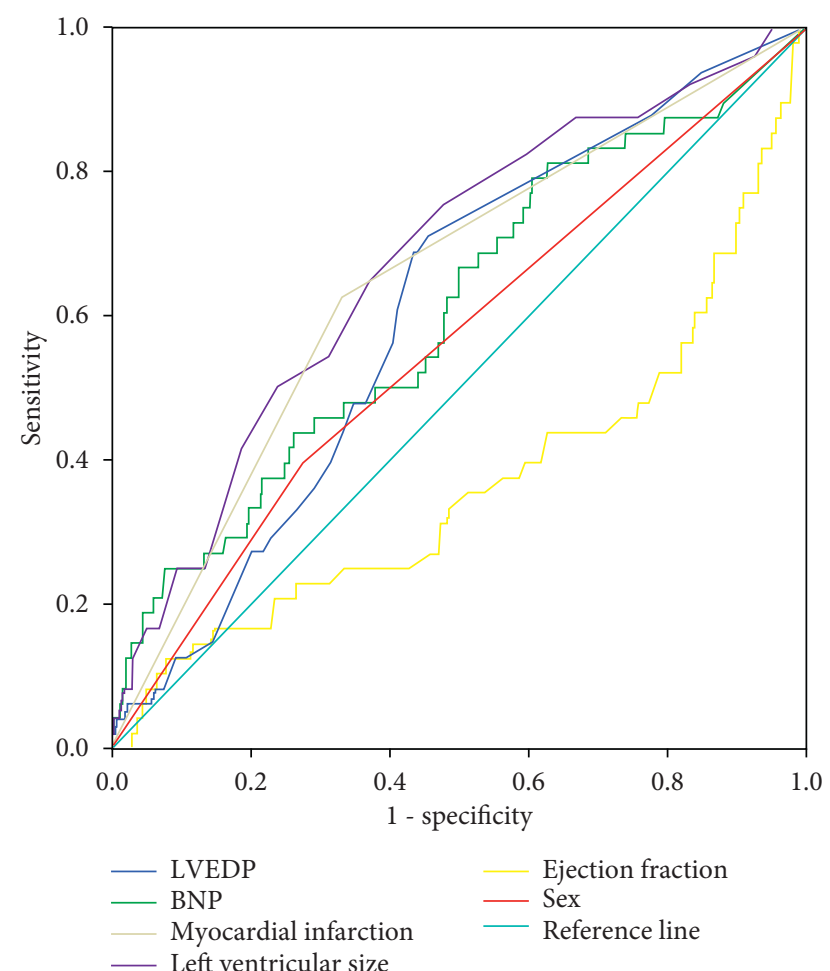

FIGURE 1: The area under the curve for the predictive performance of different indexes for contrast-induced nephropathy incidence in the cohort. BNP, B-type natriuretic peptide; LVEDP, left ventricular end-diastolic pressure.

according to these different views, our study aimed to identify indicators that could guide precise hydration treatment and to serve as a guide for the protective administration of furosemide to prevent CIN.

Accurate evaluation of blood volume status is the key step in optimizing hydration therapy. The LVEDP, which can accurately reflect the left ventricular preload, has been used as the evaluation index. A single-center, retrospective study has shown that LVEDP is an independent predictor of CIN (OR, 3.41; 95\% CI, 2.34-4.99), and the incidence of CIN in patients with LVEDP $\geq 20 \mathrm{mmHg}(19.5 \%)$ significantly increases [12]. Moreover, for every $5 \mathrm{mmHg}$ increase in LVEDP, the risk of CIN increases by 1.26 times. Several studies have reported that intervention based on LVEDP can significantly reduce the incidence of CIN and major adverse events (all-cause mortality, myocardial infarction, and longterm hemodialysis) at late follow-up [13, 14]. An increase in LVEDP values beyond the normal range indicates a change in left ventricular pressure, which leads to an increase in BNP release. Stolker and Rich [15] have pointed out that BNP level is an independent predictor of increased LVEDP in elderly patients undergoing diagnostic cardiac catheterization, and a BNP level increase can increase the estimated LVEDP value [16]. Therefore, the combined monitoring of LVEDP and BNP levels to evaluate the degree of hydration can inform individualized diuretic plans to improve clinical accuracy. In particular, compared with LVEDP, BNP is relatively easy to obtain.
4.1. Study Limitations. In interpreting these data, some limitations should be considered. No long-term follow-up study was conducted on these patients, and the long-term influence of the guidance program could not be obtained.

\section{Conclusion}

It is crucial to evaluate the LVEDP and BNP levels to optimize the administration of furosemide during PCI. Our study showed that an LVEDP value of $15.5 \mathrm{mmHg}$ and a BNP level of $116.5 \mathrm{pg} / \mathrm{mL}$ were the optimal cut-off values to predict the occurrence of CIN. The administration of furosemide after the assessment of effective blood volume can further reduce the occurrence of CIN, which indicates the effectiveness of individualized precision treatment schemes and is worthy of further promotion in clinical practice.

\section{Data Availability}

The data that support the findings of this study are available from the corresponding author upon reasonable request.

\section{Disclosure}

The funding sources had no roles in the study design; in the collection, analysis, or interpretation of data; in the writing of the report; or in the decision to submit the article for publication.

\section{Conflicts of Interest}

The authors declare that there are no conflicts of interest regarding the publication of this article.

\section{Acknowledgments}

This study was supported by the Natural Science Foundation of Hebei Province (grant nos. H2018206066 and H2020206409); the Chinese Cardiovascular AssociationAccess fund (grant no. 2018-CCA-AF-007); and the China Cardiovascular Association-Cardiac Rehabilitation and Metabolic Therapy Research fund (grant no. CCA-CRMT1806). The authors thank all the study participants for their time and for providing valuable data. The authors also thank all faculty members from the Department of Cardiology, Second Hospital of Hebei Medical University, for their enduring support, assistance, and valuable comments on the design of this study.

\section{Supplementary Materials}

Supplementary Table S1: Comparison of renal function between the experimental and control groups. Supplementary Table S2: Logistic regression analysis was performed to further explore the predictive value of LVEDP and BNP for CIN. CONSORT checklist: CONSORT 2010 checklist of information to include when reporting a randomized trial. . (Supplementary Materials) 


\section{References}

[1] R. Rear, R. M. Bell, and D. J. Hausenloy, "Contrast-induced nephropathy following angiography and cardiac interventions," Heart, vol. 102, no. 8, pp. 638-648, 2016.

[2] M. A. Hossain, E. Costanzo, J. Cosentino et al., "Contrastinduced nephropathy: pathophysiology, risk factors, and prevention," Saudi Journal of Kidney Diseases and Transplantation, vol. 29, pp. 1-9, 2018.

[3] R. Morcos, M. Kucharik, P. Bansal et al., "Contrast-induced acute kidney injury: review and practical update," Clinical Medicine Insights: Cardiology, vol. 13, Article ID 1179546819878680, 2019.

[4] G.-Q. Gu, R. Lu, W. Cui et al., "Low-dose furosemide administered with adequate hydration reduces contrast-induced nephropathy in patients undergoing coronary angiography," Cardiology, vol. 125, no. 2, pp. 69-73, 2013.

[5] G. Gu, H. Xing, Y. Zhou, and W. Cui, "Inverse correlation between left ventricular end-diastolic pressure and contrastinduced nephropathy in patients undergoing percutaneous coronary intervention," Clinical and Experimental Nephrology, vol. 22, no. 4, pp. 808-814, 2018.

[6] S. Masson, R. Latini, I. S. Anand et al., "Direct comparison of B-type natriuretic peptide (BNP) and amino-terminal proBNP in a large population of patients with chronic and symptomatic heart failure: the valsartan Heart Failure (ValHeFT) data," Clinical Chemistry, vol. 52, no. 8, pp. 1528-1538, 2006.

[7] Y. Isaka, H. Hayashi, H. Hayashi et al., "Guideline on the use of iodinated contrast media in patients with kidney disease 2018," Japanese Journal of Radiology, vol. 38, no. 1, pp. 3-46, 2020.

[8] K. M. Ho and B. M. Power, "Benefits and risks of furosemide in acute Kidney injury," Anaesthesia, vol. 65, no. 3, pp. 283-293, 2010.

[9] A. Putzu, M. Boscolo Berto, A. Belletti et al., "Prevention of contrast-induced acute Kidney injury by furosemide with matched hydration in patients undergoing interventional procedures," JACC: Cardiovascular Interventions, vol. 10, no. 4, pp. 355-363, 2017.

[10] J.-M. Weinstein, S. Heyman, and M. Brezis, "Potential deleterious effect of furosemide in radiocontrast nephropathy," Nephron, vol. 62, no. 4, pp. 413-415, 1992.

[11] N. Duan, J. Zhao, Z. Li et al., "Furosemide with saline hydration for prevention of contrast-induced nephropathy in patients undergoing coronary angiography: a meta-analysis of randomized controlled trials," Medical Science Monitor: International Medical Journal of Experimental and Clinical Research, vol. 21, pp. 292-297, 2015.

[12] G. Wu, C. Liu, and Q. Zhang, "The predicted value of left ventricular diastolic period pressure on coronary artery intervention in patients with aneurysmal nephropathy (article in Chinese)," Chinese Journal of Arteriosclerosis, vol. 27, pp. 874-880, 2019.

[13] S. S. Brar, V. Aharonian, P. Mansukhani et al., "Haemodynamic-guided fluid administration for the prevention of contrast-induced acute kidney injury: the POSEIDON randomised controlled trial," The Lancet, vol. 383, no. 9931, pp. 1814-1823, 2014.

[14] A. Bagai, P. W. Armstrong, A. Stebbins et al., "Prognostic implications of left ventricular end-diastolic pressure during primary percutaneous coronary intervention for ST-segment elevation myocardial infarction: findings from the Assessment of Pexelizumab in Acute Myocardial Infarction study," American Heart Journal, vol. 166, no. 5, pp. 913-919, 2013.

[15] J. M. Stolker and M. W. Rich, "Clinical utility of B-type natriuretic peptide for estimating left ventricular filling pressures in unselected elderly patients undergoing diagnostic coronary angiography," Journal of Invasive Cardiology, vol. 22, pp. 107-112, 2010.

[16] S. Niizuma, Y. Iwanaga, T. Washio et al., "Clinical significance of increased cardiac troponin $\mathrm{T}$ in patients with chronic hemodialysis and cardiovascular disease: comparison to B-type natriuretic peptide and A-type natriuretic peptide increase," Kidney and Blood Pressure Research, vol. 44, no. 5, pp. 1050-1062, 2019. 